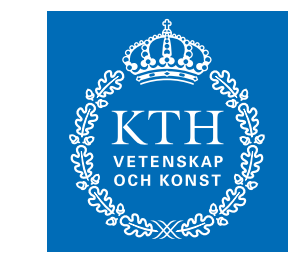

KTH Electrical Engineering

\title{
Interference Alignment over a Combination of Space and Frequency
}

IEEE International Conference on Communications 2013: IEEE ICC'13 - Workshop Beyond LTE-A.

9 June 2013, Budapest, Hungary

(C) 2013 IEEE. Personal use of this material is permitted. However, permission to reprint/republish this material for advertising or promotional purposes or for creating new collective works for resale or redistribution to servers or lists, or to reuse any copyrighted component of this work in other works must be obtained from the IEEE.

RASMUS BRANDT, PER ZETTERBERG, MATS BENGTSSON

Stockholm 2013

Signal Processing Lab

School of Electrical Engineering

KTH Royal Institute of Technology 


\title{
Interference Alignment over a Combination of Space and Frequency
}

\author{
Rasmus Brandt, Per Zetterberg, Mats Bengtsson \\ ACCESS Linnæus Center, Signal Processing Lab \\ KTH Royal Institute of Technology, Stockholm, Sweden
}

\begin{abstract}
Time or frequency extensions are integral in most information theoretic studies of interference alignment (IA), but a large majority of the more practically oriented studies have focused on narrowband space-only schemes. As wideband systems are now common, it is natural to investigate IA for frequency extended multiple antenna systems where precoding is performed over a combination of space and frequency dimensions. For this setting, we derive a necessary condition on IA feasibility using the properness framework and investigate the sum rate performance using simulations. Applying frequency extensions to multiple antenna systems allows for some additional users to be served interference-free, but our numerical results with synthetic channels indicate a practically more important improvement in terms of a power gain. Emulating a particular scenario using channel measurements, with real-world path losses and channel correlations, we see similar performance gains.
\end{abstract}

\section{INTRODUCTION}

Interference management is critical for the performance of multiuser networks [1]. Traditional approaches to mitigating the negative impact of interference include treating the interference as noise when it is weak, or decoding and removing it using successive interference cancellation when it is strong. In the intermediate regime, when the interference power is on the order of the signal power, users are typically orthogonalized in some dimension, e.g. time or frequency. In this intermediate regime, the idea of interference alignment (IA) [2] has lately garnered a large amount of attention.

Interference alignment can be achieved using linear transmit filters, by aligning all interference in lower dimensional subspaces at all receivers simultaneously. The interference can then be removed by linear zero-forcing filters. By maximizing the number of interference-free data streams in the network, a metric called the sum degrees of freedom (sum DoF), the asymptotic slope of the sum rate curve is also maximized.

Pure interference alignment, corresponding to completely removing all inter-user interference, is not the be-all and endall of interference management in practical networks. Noise, limited channel state information, transceiver impairments and other issues may lead to other methods being better options for implementation. IA is still interesting, since it gives insight into how practical algorithms should work at high signal-to-noise ratio (SNR), where the network performance is fundamentally interference limited.

For a given number of transmitted data streams, the feasibility of IA depends on the signal space dimensions available. Either spatial dimensions [3], [4] or time/frequency dimensions [2] can be used for IA. In this paper, we investigate interference alignment for multiple-input multiple-output orthogonal frequency-division multiplexing (MIMO-OFDM) systems [5], where joint transmission over a combination of space and frequency dimensions is possible. The combination of space and frequency has previously been used in MIMO-OFDM systems for improving diversity using space-frequency block codes [6], but its use for increasing the signal dimensions for IA precoding has not received a lot of attention so far.

The feasibility of IA for single-antenna OFDM systems was studied in [7], where a necessary condition on feasibility was derived. An evaluation of practical performance was performed in [8], where practical methods related to IA performed well when applied to measurements from an urban macrocell scenario. Investigations of IA for MIMO-OFDM systems have previously been reported in [9], [10], where testbeds were used to show the real-world feasibility of IA. In both studies, the subcarriers were used independently. In [11], loose outer bounds for the feasibility of space-frequency precoding were proposed for channels with limited diversity.

In this paper, we derive a necessary condition for IA feasibility over frequency extended channels using the properness framework of [3]. We investigate the implications of joint space-frequency precoding and evaluate performance for IA and related schemes using simulations. For an investigated scenario with synthetic channels, both a sum DoF gain as well as a substantial power gain can be achieved by employing space-frequency precoding instead of space-only precoding over the subcarriers. For measured channels with real-world path losses and channel correlations, the same effects from employing space-frequency precoding are observed.

\section{SYSTEM MODEL}

We assume a MIMO-OFDM system with $M_{r}$ receive antennas, $M_{t}$ transmit antennas and $N_{c}$ subcarriers. There are $K$ transmitter-receiver pairs ('users'), and we denote the channel matrix at the $n$th subcarrier between transmitter $l$ and receiver $k$ as $\mathbf{H}_{k l}^{(n)} \in \mathbb{C}^{M_{r} \times M_{t}}$. The channels at different subcarriers are orthogonal due to the usage of OFDM, but may be correlated depending on the coherence bandwidth of the channel. In order to transmit jointly over the available space and frequency dimensions, we form the frequency extended channel matrix

$$
\mathbf{H}_{k l}=\operatorname{blkdiag}\left(\mathbf{H}_{k l}^{(1)}, \ldots, \mathbf{H}_{k l}^{\left(N_{c}\right)}\right) \in \mathbb{C}^{M_{r} N_{c} \times M_{t} N_{c}}, \quad \forall k, l
$$

which is block-diagonal due to the orthogonality between subcarriers. This structure will show to be important when analyzing the feasibility of IA for joint space-frequency precoding in the next section. 
Our model for the multiuser interaction is the interference channel, and hence the input-output relationship is

$$
\mathbf{y}_{k}=\mathbf{H}_{k k} \mathbf{V}_{k} \mathbf{x}_{k}+\sum_{\substack{l=1 \\ l \neq k}}^{K} \mathbf{H}_{k l} \mathbf{V}_{l} \mathbf{x}_{l}+\mathbf{z}_{k}, \quad \forall k
$$

where the channel matrices $\mathbf{H}_{k l}$ are independent between links. We let $\mathbf{x}_{k} \in \mathbb{C}^{d}$ be the signal sent from transmitter $k$ intended for receiver $k$, linearly precoded by $\mathbf{V}_{k} \in \mathbb{C}^{M_{t} N_{c} \times d}$. We assume that all users are served $d$ data streams and that $\left\|\mathbf{V}_{k}\right\|_{F}^{2} \leq P_{k}$. We assume circularly symmetric complex white Gaussian noise $\mathbf{z}_{k} \sim \mathcal{C N}\left(\mathbf{0}_{M_{r} N_{c} \times 1}, \sigma^{2} \mathbf{I}_{M_{r} N_{c}}\right)$. The data rate with an optimal single-user detector for user $k$, assuming a Gaussian codebook $\mathbf{x}_{k} \sim \mathcal{C N}\left(\mathbf{0}_{d \times 1}, \mathbf{I}_{d}\right)$ and neglecting the cyclic prefix, is

$$
R_{k}=\log _{2} \operatorname{det}\left(\mathbf{I}_{M_{r}}+\mathbf{Q}_{k}^{-1} \mathbf{H}_{k k} \mathbf{V}_{k} \mathbf{V}_{k}^{H} \mathbf{H}_{k k}^{H}\right)
$$

where $\mathbf{Q}_{k}=\sigma^{2} \mathbf{I}_{M_{r}}+\sum_{l \neq k} \mathbf{H}_{k l} \mathbf{V}_{k} \mathbf{V}_{k}^{H} \mathbf{H}_{k k}^{H}$ is the interference and noise covariance matrix.

If linear filters $\mathbf{U}_{k} \in \mathbb{C}^{M_{r} N_{c} \times d}$ are employed at the receiver side, the resulting channel is

$$
\widetilde{\mathbf{x}_{k}}=\mathbf{U}_{k}^{H} \mathbf{y}_{k}=\underbrace{\mathbf{U}_{k}^{H} \mathbf{H}_{k k} \mathbf{V}_{k}}_{\text {effective channel }} \mathbf{x}_{k}+\underbrace{\mathbf{U}_{k}^{H} \sum_{l \neq k} \mathbf{H}_{k l} \mathbf{V}_{l} \mathbf{x}_{l}}_{\text {residual interference }}+\underbrace{\mathbf{U}_{k}^{H} \mathbf{z}_{k}}_{\text {filtered noise }}
$$

Interference alignment corresponds to making sure that the residual interference is identically zero at all receivers, while maintaining the rank of the effective channel:

$$
\begin{aligned}
\mathbf{U}_{k}^{H} \mathbf{H}_{k l} \mathbf{V}_{l} & =\mathbf{0}_{d}, & & \forall k \neq l \\
\operatorname{rank}\left(\mathbf{U}_{k}^{H} \mathbf{H}_{k k} \mathbf{V}_{k}\right) & =d, & & \forall k .
\end{aligned}
$$

Any solution to (2)-(3) does indeed give optimal sum rate scaling at high SNR. Practical resource allocation algorithms should however not try to solve (2)-(3), but rather try to optimize the user rates in (1).

\section{FEASIBILITy OF INTERFERENCE AlignMENT FOR JOINT SPACE-FREQUENCY PRECODING}

The feasibility of IA for multiuser MIMO-OFDM systems is determined by the solvability of (2)-(3). Similarly to [7], we will in the following assume that the receive and transmit filters are marginally isotropic, such that (3) will be satisfied with probability 1 . We also assume no particular structure on the $\mathbf{V}_{k}$, meaning that all space and frequency dimensions can be used for joint precoding.

We focus on the solvability of (2), which is a set of bilinear equations in the components of $\mathbf{U}_{k}$ and $\mathbf{V}_{k}$. The solvability of this type of polynomial equations is typically analyzed using techniques from algebraic geometry. Here, similar to the approach in [7], we use the properness framework of [3] and Bernstein's theorem in algebraic geometry [12, Ch. 7].

For the symmetric scenario at hand, the concept of properness amounts to having $N_{v} \geq N_{e}$, where $N_{v}$ is the number of complex variables in (2) and $N_{e}$ is the number of equations in (2). In [3], properness was shown to be necessary and sufficient for IA feasibility for single-carrier MIMO systems with $d=1$. Here, we will show a necessary condition for $d=1$ for the frequency extended MIMO channel.
We first derive the properness condition for arbitrary $d$. The number of equations in (2) is $N_{e}=K d^{2}(K-1)$. In order to count the number of complex variables in (2), we need to use a parametrization with the fewest number of independent variables possible. In total, there are $K\left(M_{r}+M_{t}\right) N_{c} d$ coefficients in all filters. However, the number of independent variables in the polynomial system is lower.

First, we notice that only $\operatorname{span}\left(\mathbf{U}_{k}\right)$ and $\operatorname{span}\left(\mathbf{V}_{l}\right)$ matters in order to satisfy (2). There is therefore no loss in letting

$$
\mathbf{U}_{k}=\left(\begin{array}{c}
\mathbf{I}_{d} \\
\overline{\mathbf{U}}_{k}
\end{array}\right), \quad \mathbf{V}_{k}=\left(\begin{array}{c}
\mathbf{I}_{d} \\
\overline{\mathbf{V}}_{k}
\end{array}\right),
$$

which removes $2 K d^{2}$ coefficients. For some non-zero complex numbers $\beta^{(n)}$, let $\boldsymbol{\beta}_{U}=\operatorname{blkdiag}\left(\beta^{(1)} \mathbf{I}_{M_{r}}, \ldots, \beta^{\left(N_{c}\right)} \mathbf{I}_{M_{r}}\right)$ and $\boldsymbol{\beta}_{V}=\operatorname{blkdiag}\left(\beta^{(1)} \mathbf{I}_{M_{t}}, \ldots, \beta^{\left(N_{c}\right)} \mathbf{I}_{M_{t}}\right)$. Then notice that

$$
\begin{aligned}
0 & =\mathbf{U}_{k}^{H} \mathbf{H}_{k l} \mathbf{V}_{l}=\mathbf{U}_{k}^{H} \boldsymbol{\beta}_{U}{ }^{-1} \boldsymbol{\beta}_{U} \mathbf{H}_{k l} \mathbf{V}_{l} \\
& =\mathbf{U}_{k}^{H} \boldsymbol{\beta}_{U}{ }^{-1} \mathbf{H}_{k l} \boldsymbol{\beta}_{V} \mathbf{V}_{l}={\widetilde{\mathbf{U}_{k}}}^{H} \mathbf{H}_{k l} \widetilde{\mathbf{V}_{l}}
\end{aligned}
$$

where the third equality is due to the block-diagonal structure of $\boldsymbol{\beta}_{U}, \boldsymbol{\beta}_{V}$ and $\mathbf{H}_{k l}$. That is, as similarly noted in [7], any solution to (2) remains a solution after the transformation

$$
\widetilde{\mathbf{U}_{k}}=\boldsymbol{\beta}_{U}{ }^{-H} \mathbf{U}_{k}, \quad \widetilde{\mathbf{V}_{l}}=\boldsymbol{\beta}_{V} \mathbf{V}_{l}, \quad \forall k, l .
$$

The condition in (3) is still satisfied after this transformation. Under this invariance, we can use the $\beta^{(n)}$ to further remove variables from the polynomial system. For instance, let

$$
\widetilde{\mathbf{V}_{1}}=\boldsymbol{\beta}_{V}\left(\begin{array}{c}
\mathbf{I}_{d} \\
\overline{\mathbf{V}}_{1}
\end{array}\right)=\left(\begin{array}{ccc}
\beta^{(1)} \mathbf{I}_{M_{t}} & & \\
& \ddots & \\
& & \beta^{\left(N_{c}\right)} \mathbf{I}_{M_{t}}
\end{array}\right)\left(\begin{array}{c}
\mathbf{I}_{d} \\
\overline{\mathbf{V}}_{1}
\end{array}\right) .
$$

Since the topmost $d$ rows of $\widetilde{\mathbf{V}_{1}}$ and $\widetilde{\mathbf{U}_{1}}$ should not be altered, we can fix $N_{c}-\left\lceil\frac{d}{\min \left(M_{r}, M_{t}\right)}\right\rceil$ of the coefficients in $\widetilde{\mathbf{V}_{1}}$ by selecting the $\beta^{(n)}$ appropriately. Subsequently, the number of variables in the polynomial system (2) is $N_{v}=$ $K\left(M_{r}+M_{t}\right) N_{c} d-2 K d^{2}-\left(N_{c}-\left\lceil\frac{d}{M_{\min }}\right\rceil\right)$ and the properness condition $N_{v} \geq N_{e}$ then corresponds to

$K d\left(\left(M_{r}+M_{t}\right) N_{c}-2 d\right)-K d^{2}(K-1)-\left(N_{c}-\left\lceil\frac{d}{M_{\min }}\right\rceil\right) \geq 0$.

where $M_{\min }=\min \left(M_{r}, M_{t}\right)$. Although it is not obvious if Bernstein's theorem can be used to show that (4) is a necessary condition for IA feasibility, our numerical tests suggest that it is sufficient.

To get a necessary condition for IA feasibility, we specialize to the $d=1$ case. After rearranging terms and noting that $K$ is an integer, the properness condition (4) is $K \leq\left\lfloor\left(M_{r}+M_{t}\right) N_{c}-1-\frac{N_{c}-1}{K}\right\rfloor$ which is equivalent to

$$
K \leq \begin{cases}\left(M_{r}+M_{t}\right)-1 & N_{c}=1 \\ \left(M_{r}+M_{t}\right) N_{c}-2 & N_{c} \geq 2\end{cases}
$$

if $K \geq N_{c}$. To see why (5) is necessary for IA feasibility when $\bar{d}=1$, we use an argument previously used in [3] and [7]. Assume that (5) does not hold and thus $N_{v}<N_{e}$. Then we can eliminate $N_{e}-N_{v}$ equations to end up with a $N_{v}$ by $N_{v}$ polynomial system of equations. Through Bernstein's theorem [12, Ch. 7], we know that this polynomial system has 
a bounded number of solutions. Since the coefficients in the eliminated $N_{e}-N_{v}$ equations are random variables independent of the coefficients in the reduced polynomial system, any solution to this system will not satisfy the eliminated equations with probability 1 . Hence, (5) is a necessary condition on IA feasibility.

The condition in (5) subsumes the condition in [7], and the necessary part of the condition in [3]. From the feasibility perspective, space and frequency dimensions are equivalent, save for a constant loss of 1 when using a frequency extended channel instead of a single-carrier system.

\section{SPACE-Frequency Precoding VS. SPACE-ONLY PRECODING}

In the previous section, we assumed that all subcarriers in the frequency extended channel were used for joint transmission. We call this case fully joint space-frequency precoding. This may not always be practical, or even wanted, in a real system. Instead, by introducing some structure into $\mathbf{V}_{k}$, orthogonal subcarrier groups can be formed. Parallel IA operations can then be multiplexed over the orthogonal subcarrier groups.

Grouped joint space-frequency precoding can be performed by forming $N_{g}$ groups, each containing $N_{f}=N_{c} / N_{g}$ subcarriers. One particular way of placing the subcarriers into groups is equidistant grouping [8]. Each group contains subcarriers selected equidistantly, while adjacent subcarriers belong to different groups. For example, with $N_{c}$ subcarriers, $N_{g}$ groups and one data stream per group, the precoders would look like:

$$
\mathbf{V}_{k}^{\text {equidistant }}=\left(\begin{array}{c}
\operatorname{blkdiag}\left(\mathbf{v}_{k, 1}^{(1)}, \mathbf{v}_{k, 2}^{(2)}, \ldots, \mathbf{v}_{k, N_{g}}^{\left(N_{g}\right)}\right) \\
\operatorname{blkdiag}\left(\mathbf{v}_{k, 1}^{\left(N_{g}+1\right)}, \mathbf{v}_{k, 2}^{\left(N_{g}+2\right)}, \ldots, \mathbf{v}_{k, N_{g}}^{\left(2 N_{g}\right)}\right) \\
\vdots \\
\operatorname{blkdiag}\left(\mathbf{v}_{k, 1}^{\left(N_{c}-N_{g}+1\right)}, \mathbf{v}_{k, 2}^{\left(N_{c}-N_{g}+2\right)}, \ldots, \mathbf{v}_{k, N_{g}}^{\left(N_{c}\right)}\right)
\end{array}\right) .
$$

Another way of forming subcarrier groups is space-only precoding, where $N_{g}=N_{c}$. For this case, there is no coupling between the precoding over different subcarriers.

\section{A. Gain from Fully Joint Space-Frequency Precoding}

For a frequency extended channel with space-only precoding, the feasibility condition (5) gives that the maximum number of single-stream users that can partake in IA operation per subcarrier is $K_{1}^{\mathrm{max}}=M_{r}+M_{t}-1$. By performing fully joint space-frequency precoding, $K_{N_{c}}^{\max }=\left(M_{r}+M_{t}\right) N_{c}-2$ single-stream users can be served. More interference-free data streams can be transmitted with fully joint space-frequency precoding if $K_{N_{c}}^{\max }>N_{c} K_{1}^{\max }$. Rearranging, this becomes

$$
\left(M_{r}+M_{t}\right) N_{c}-2>\left(M_{r}+M_{t}\right) N_{c}-N_{c} \quad \Leftrightarrow \quad N_{c} \geq 3 .
$$

Fig. 1 gives an illustration of this condition. Starting from $N_{c}=3$, fully joint space-frequency precoding is able to accommodate more users than space-only precoding. For $M_{r}=M_{t}=2$, at least $K=10$ is needed to see a benefit from using the frequency extensions for space-frequency precoding. Similarly for $M_{r}=M_{t}=3, K=16$ is needed.

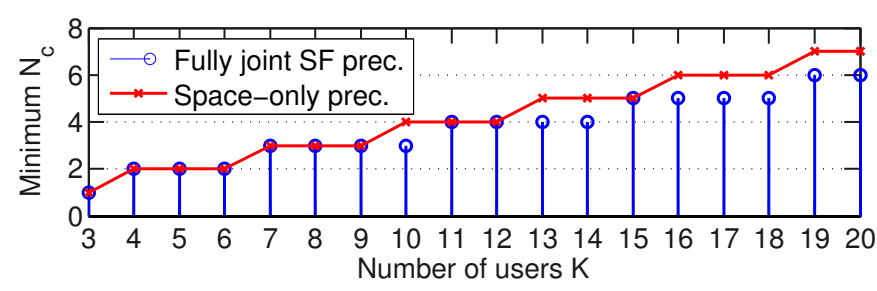

(a) Number of antennas $M_{r}=M_{t}=2$

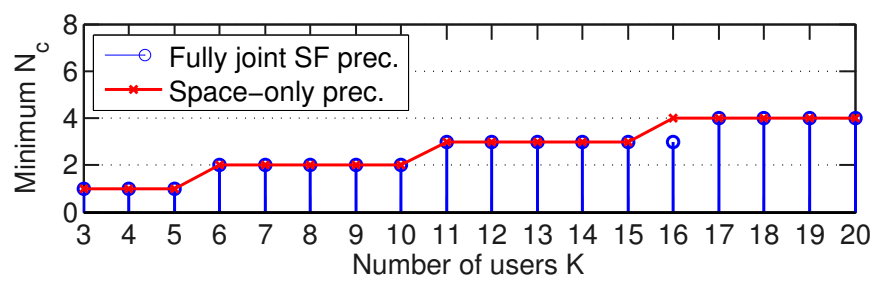

(b) Number of antennas $M_{r}=M_{t}=3$

Fig. 1. Min. number of subcarriers needed for serving $K$ single-stream users (i.e. $d=1$ ), using fully joint space-freq. precoding or space-only precoding.

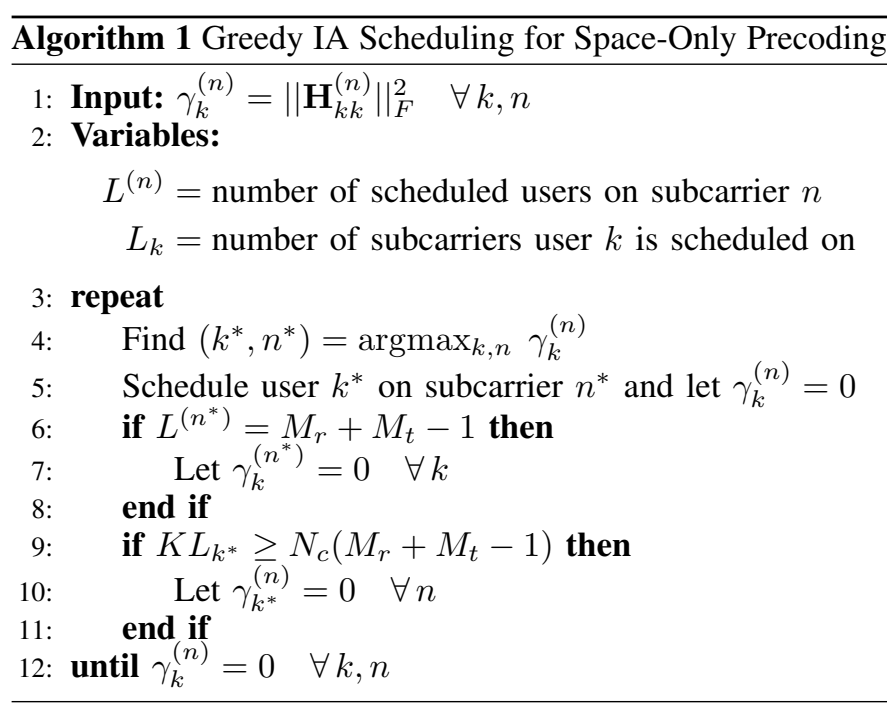

\section{B. Scheduling for Space-Only Precoding}

If fully joint space-frequency precoding is employed, scheduling is not needed since all users in the network can be active over all subcarriers. If space-only precoding is used however, different subcarriers might potentially serve different user combinations. A scheduler should pick out what users that are favourable for being served together. At the same time, the scheduler should ensure that the IA feasibility conditions are not violated, and that some form of fairness is maintained between users. Since the scheduling problem is combinatorial, and hence hard to solve to optimality, we propose a simple greedy approach in Algorithm 1. The scheduling metric is the direct channel strength, whereas a more elaborate scheduler should take into account the strength of the cross-links, which users are spatially compatible for being served at the same time, as well as the long-term performance of the users.

\section{Numerical Results}

For the numerical performance investigations, we use the sum rate $\sum_{k=1}^{K} R_{k}$ as the performance metric. To find the 


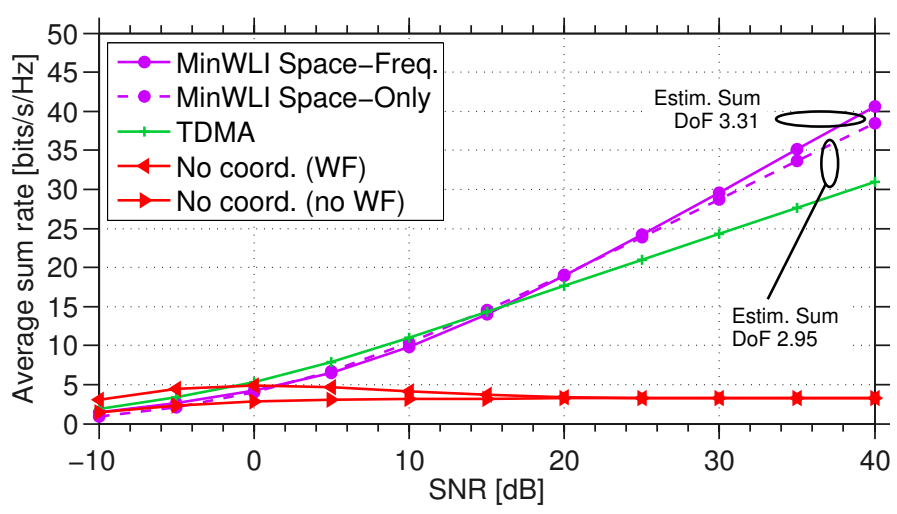

(a) Performance of pure IA solution from MinWLI

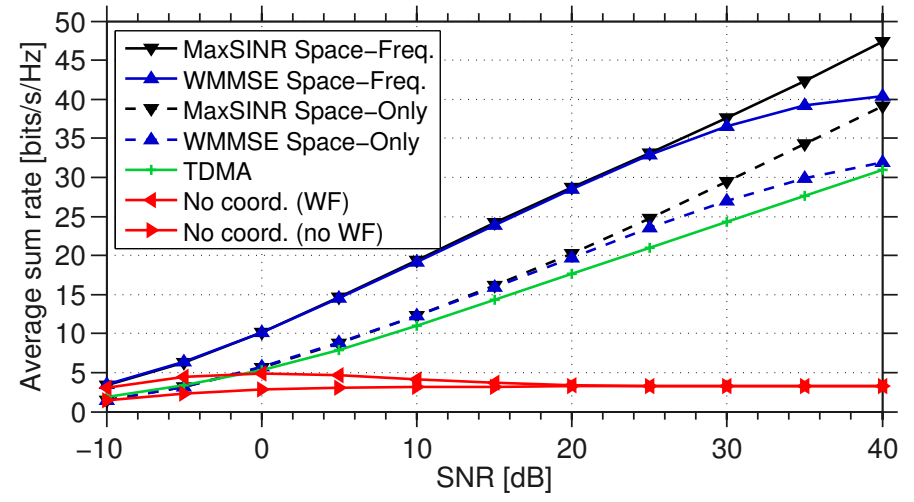

(b) Performance of practical resource allocation schemes

Fig. 2. Performance for synthetic channel with $K=10, M_{r}=M_{t}=2$ and $N_{c}=30$. For space-frequency precoding, the subcarriers are split into $N_{g}=10$ groups such that each group contains $N_{f}=3$ subcarriers. All users are served one data stream in all groups, i.e. the maximum sum DoF is $K / N_{f}=3.33 \ldots$. For space-only precoding with scheduling, 3 single-stream users are accommodated per subcarrier, giving a maximum sum DoF of 3.

precoders $\mathbf{V}_{k}$, we use the by now almost standard distributed IA method MinWLI and ad-hoc stream signal-to-interferenceand-noise ratio maximizing method MaxSINR from [13]. Furthermore, we apply a method which tries to maximize the sum rate by transforming the optimization problem into a weighted MMSE problem, which is solved by block coordinate descent [14]. We will call this method MaxRate (WMMSE). All methods are applied to 100 channel realizations. For convergence, we let MinWLI run until the relative interference leakage $\operatorname{Tr}\left(\sum_{k} \sum_{l \neq k} \mathbf{U}_{k}^{H} \mathbf{H}_{k l} \mathbf{V}_{l} \mathbf{V}_{l}^{H} \mathbf{H}_{k l}^{H} \mathbf{U}_{k}\right) / \operatorname{Tr}\left(\sum_{k, l} \mathbf{H}_{k l} \mathbf{V}_{l} \mathbf{V}_{l}^{H} \mathbf{H}_{k l}^{H}\right)$ is less than $10^{-9}$. Since it is not guaranteed to converge, we let MaxSINR run for 2000 iterations. This seems to be sufficient for good high-SNR performance. Finally, MaxRate (WMMSE) is run until the relative change in sum rate is less than $10^{-5}$. All methods are initialized with truncated DFT matrices. As performance benchmarks, we use time-division multiple access (TDMA) and uncoordinated transmission over the transmitters with and without water filling.

We will use both synthetic and measured channels for evaluating the performance for a $K=10, M_{r}=M_{t}=2$ scenario, since this is the first case where theoretical gains are possible according to Fig. 1a. The system has $N_{c}=30$ subcarriers, and we compare the performance of space-frequency precoding with equidistant grouping and $N_{g}=10$ groups to the performance of space-only precoding with scheduling. The power is allocated uniformly over groups.

Both the equidistant grouping and the greedy scheduling are in general suboptimal for the allocation of users into groups and onto subcarriers. The greedy scheduling might however perform relatively better when scenarios with heterogeneous path losses are studied, as will be seen in Section V-B. We leave the improvement of the grouping method for future work.

\section{A. Synthetic Channels}

First we use a tapped delay line with $L_{t}$ taps for simulating a block fading frequency-selective channel. We assume rich scattering and an exponentially decaying power-delay profile such that the channel impulse response in one block is

$$
\mathbf{H}_{k l}[t]=c \sqrt{e^{-\frac{t}{W \tau}}} \mathbf{H}_{k l}^{w}[t], \quad t=0 \ldots L_{t}-1 .
$$

The coefficients of $\mathbf{H}_{k l}^{w}[t]$ are i.i.d. $\mathcal{C N}(0,1)$ and are constant within one transmission block, but vary independently between blocks. The channels are normalized to $\sum_{t=0}^{L_{t}-1} \mathbb{E}\left(\left\|\mathbf{H}_{k l}[t]\right\|_{F}^{2}\right)=M_{r} M_{t}$ using $c$, and we assume a bandwidth delay spread product $W \tau=1.5$ and $L_{t}=6$ taps.

The performance for $P_{k}=P$ can be seen in Fig. 2, where a large $\mathrm{SNR}=P /\left(N_{c} \sigma^{2}\right)$ range is shown in order to illustrate the scaling at high SNR. The expected gain in high-SNR slope from space-frequency precoding over spaceonly precoding is present for the pure IA solution provided by MinWLI, but the performance gain in terms of sum rate is small. Another effect that can be seen in Fig. 2 is the large power gains for MaxSINR when employing space-frequency precoding. Although not IA gains per se, they signify that IA solutions with significantly higher sum rate can be found when the signal space dimension is increased by combining space and frequency. In this particular case, space-frequency precoding with MaxSINR considerably outperforms TDMA.

\section{B. Measured Channels}

In order to evaluate performance for real-world path losses and channel correlations, we use channel measurements [15], [16]. The measurements were taken along a $70 \mathrm{~m}$ long office building corridor (see [15] for map) using an $20 \mathrm{MHz}$ LTEAdvanced testbed with a carrier frequency of $2.7 \mathrm{GHz}$. The measurement antenna array consisted of four dual-polarized patch elements linearly arranged with $0.5 \lambda$ spacing. Measurements were taken from three 8-antenna base stations (BSs), one located in the middle of the corridor (square, $2.3 \lambda$ antenna spacing), one located at the end of the corridor (square, $1.15 \lambda$ horizontal spacing, $1.85 \lambda$ vertical spacing) and one located $65 \mathrm{~m}$ away on an outdoor wall facing the corridor (linear, $1.15 \lambda$ antenna spacing). The channels were measured in 100 frequency points, out of which we select 30 equidistant points.

In order to emulate a $K=10$ scenario, we select ten spatially separated measurement route segments, similar to the procedure in [8]. We select one dual-polarized patch antenna from the receive array (i.e. $M_{r}=2$ ), all antennas from the first BS and 6 antennas from the two other BSs. To achieve a $M_{t}=2$ scenario, the selected BS antennas are 


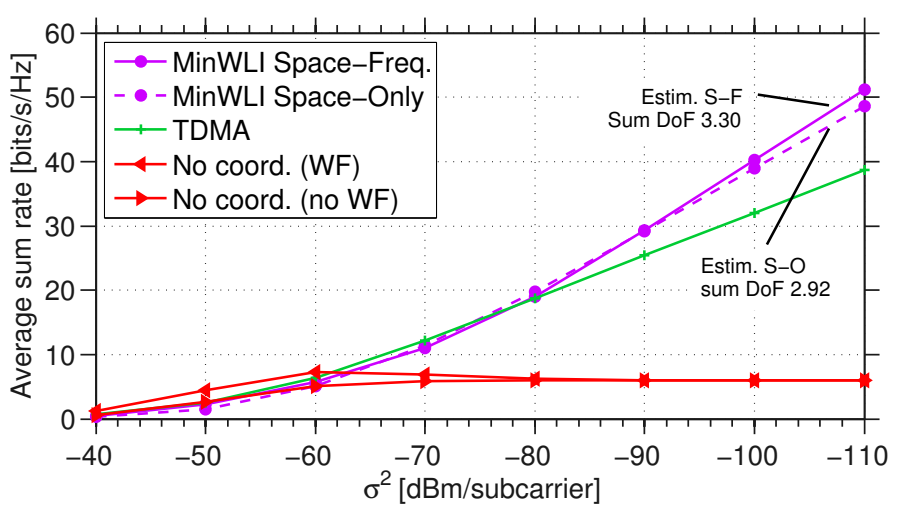

(a) Performance of pure IA solution from MinWLI

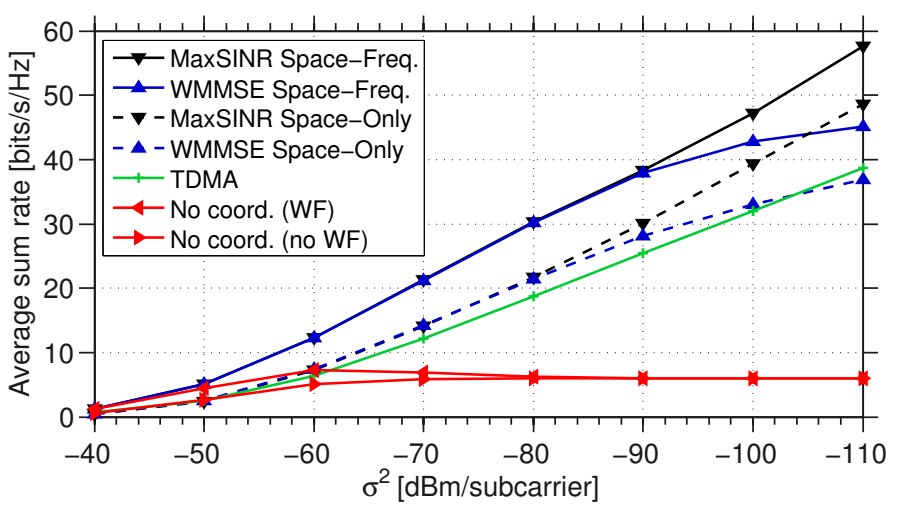

(b) Performance of practical resource allocation schemes

Fig. 3. Performance for the same parameters as in Fig. 2, emulated using channel measurements instead of synthetic channels.

split into 10 virtual 2-antenna transmitters. The simulated transmit powers $P_{k}$ were $6 \mathrm{dBm}$ for the virtual transmitters corresponding to the indoor central $\mathrm{BS}, 10 \mathrm{dBm}$ for the virtual transmitters corresponding to the indoor peripheral $\mathrm{BS}$ and $30 \mathrm{dBm}$ for the virtual transmitters corresponding to the outdoor BS. These transmit powers were selected such that the signal-to-interference ratios of the emulated cross-links were approximately $0-15 \mathrm{~dB}$. In order to simulate varying SNRs, we vary the noise power $\sigma^{2}$, which is the same for all receivers.

For the pure IA solution with space-frequency precoding in Fig. 3a, a high-SNR slope similar to the one experienced with the synthetic channel is present. The sum rate of the space-only solution is however slightly higher at intermediate SNR, which may be due to the greedy scheduler consistently allocating fewer data streams to the user with the weakest direct channel. The space-frequency scheme always serves all users, and is therefore at a small disadvantage. In Fig. 3b, the practical resource allocation methods have been applied to the measured channels. The same type of power gain from employing space-frequency precoding that was noticeable for the synthetic channel, can also be seen when the practical methods are applied to the measured channels.

\section{CONCLUSIONS}

Based on promising prior theoretical work on interference alignment for multiple antenna systems, it is natural to investigate the combined use of multiple antennas and frequency extensions with the aim of further sum rate gains for interference channels. We have analyzed the feasibility of such systems and using simulations investigated the performance of interference alignment as well as more practical schemes. Although we only investigate one particular scenario numerically, our results indicate that the main practical advantage of space-frequency precoding comes in terms of a power gain, which is evident for both synthetic and measured channels.

\section{ACKNOWLEDGEMENT}

We would like to thank Ericsson for providing the channel measurements, and Henrik Asplund at Ericsson for valuable input.
The HiATUS project acknowledges the financial support of the Future and Emerging Technologies program within FP7 for Research of the European Commission (FET Open grant number 265578).

\section{REFERENCES}

[1] D. Gesbert, S. Hanly, H. Huang, S. Shamai Shitz, O. Simeone, and W. Yu, "Multi-cell MIMO cooperative networks: A new look at interference," IEEE JSAC, vol. 28, no. 9, pp. 1380-1408, 2010.

[2] V. R. Cadambe and S. A. Jafar, "Interference alignment and degrees of freedom of the K-user interference channel," IEEE Trans. Inf. Theory, vol. 54, no. 8, pp. 3425-3441, 2008.

[3] C. Yetis, T. Gou, S. A. Jafar, and A. Kayran, "On feasibility of interference alignment in MIMO interference networks," IEEE Trans. Signal Process., vol. 58, no. 9, pp. 4771-4782, 2010.

[4] M. Razaviyayn, G. Lyubeznik, and Z.-Q. Luo, "On the degrees of freedom achievable through ia in a MIMO interference channel," IEEE Trans. Signal Process., vol. 60, no. 2, pp. 812-821, 2012.

[5] M. Jiang and L. Hanzo, "Multiuser MIMO-OFDM for next-generation wireless systems," Proc. IEEE, vol. 95, no. 7, pp. 1430-1469, 2007.

[6] W. Zhang, X. Xiang-Gen, and K. Ben Letaief, "Space-time/frequency coding for MIMO-OFDM in next generation broadband wireless systems," IEEE Trans. Wireless Commun., vol. 14, no. 3, pp. 32 -43, 2007.

[7] C. Shi, R. Berry, and M. Honig, "Interference alignment in multi-carrier interference networks," in Inform. Theory (ISIT'11), Proc. IEEE Int. Symp., 2011, pp. 26-30.

[8] R. Brandt, H. Asplund, and M. Bengtsson, "Interference alignment in frequency - a measurement based performance analysis," in Systems, Signals and Image Process. (IWSSIP'12), Proc. Int. Conf., 2012, pp. 227-230.

[9] O. El Ayach, S. W. Peters, and R. W. Heath, "The feasibility of interference alignment over measured MIMO-OFDM channels," IEEE Trans. Veh. Technol., vol. 59, no. 9, pp. 4309-4321, 2010.

[10] P. Zetterberg and N. Moghadam, "An experimental investigation of SIMO, MIMO, interference-alignment (IA) and coordinated multi-point (CoMP)," in Systems, Signals and Image Process. (IWSSIP'12), Proc. Int. Conf., 2012, pp. 211-216.

[11] L. Li, H. Jafarkhani, and S. A. Jafar, "Towards the feasibility conditions for linear interference alignment with symbol extensions: a diversity constraint," in Global Telecommun. Conf. (GLOBECOM'12), Proc. IEEE, 2012, pp. 2352-2357.

[12] D. Cox, J. Little, and D. O'Shea, Using alg. geometry. Springer, 1998.

[13] K. Gomadam, V. R. Cadambe, and S. Jafar, "A distributed numerical approach to IA and applications to wireless intererence networks," IEEE Trans. Inf. Theory, vol. 57, no. 6, pp. 3309-3322, 2011.

[14] Q. Shi, M. Razavivayn, Z. Luo, and C. He, "An iteratively weighted MMSE approach to distributed sum-utility maximization for a MIMO interfering broadcast channel," IEEE Trans. Signal Process., vol. 59, no. 9, pp. 4331-4340, 2011.

[15] K. Werner, H. Asplund, D. V. P. Figueiredo, N. Jaldén, and B. Halvarsson, "LTE-Advanced $8 \times 8$ MIMO measurements in an indoor scenario," in Ant. and Prop. (ISAP'12), Proc. Int. Symp., 2012, pp. 750-753.

[16] K. Werner, H. Asplund, B. Halvarsson, N. Jaldén, D. V. P. Figueiredo, and A. K. Kathrein, "LTE-A field measurements: $8 \times 8$ MIMO and carrier aggregation," in Veh. Tech. Conf. (VTC'13 Spring), Proc. IEEE, 2013, pp. 750-753. 